\title{
Mel-cepstral feature extraction methods for image representation
}

\author{
Serdar Çakır \\ A. Enis Çetin \\ Bilkent University \\ Department of Electrical and Electronics \\ Engineering \\ 06800, Ankara, Turkey \\ E-mail: cakir@ bilkent.edu.tr
}

\begin{abstract}
An image feature extraction method based on the twodimensional (2-D) mel cepstrum is introduced. The concept of onedimensional mel cepstrum, which is widely used in speech recognition, is extended to 2-D in this article. The feature matrix resulting from the 2-D mel-cepstral analysis are applied to the support-vector-machine classifier with multi-class support to test the performance of the mel-cepstrum feature matrix. The AR, ORL, and Yale face databases are used in experimental studies, which indicate that recognition rates obtained by the 2-D mel-cepstrum method are superior to the recognition rates obtained using 2-D principal-component analysis and ordinary image-matrixbased face recognition. Experimental results show that 2-D mel-cepstral analysis can also be used in other image feature extraction problems. (C) 2010 Society of Photo-Optical Instrumentation Engineers. [DOI: 10.1117/1.3488050]
\end{abstract}

Subject terms: 2-D mel cepstrum; cepstral features; image feature extraction; face recognition.

Paper 100240R received Mar. 25, 2010; revised manuscript received Jul. 5 , 2010; accepted for publication Jul. 26, 2010; published online Sep. 15, 2010.

\section{Introduction}

Mel-cepstral analysis is one of the most widely used feature extraction techniques in speech processing applications, including speech and sound recognition and speaker identification. The two-dimensional (2-D) cepstrum is also used in image registration and filtering applications. ${ }^{1-4}$ To the best of our knowledge, the 2-D mel cepstrum, which is a variant of the 2-D cepstrum, is not used in image feature extraction, classification, and recognition problems. The goal of this paper is to define the 2-D mel cepstrum and show that it is a viable image representation tool.

The ordinary 2-D cepstrum of a 2-D signal is defined as the inverse Fourier transform (FT) of the logarithmic spectrum of the signal, and it is computed using a 2-D fast Fourier transformation (FFT). As a result, it is independent of pixel amplitude variations or grayscale changes, which leads to robustness against illumination variations. Since it is a FT-based method, it is also independent of translational shifts. The 2-D mel cepstrum, which is based on logarithmic decomposition of a frequency-domain grid, has the same shift and amplitude invariance properties as the 2-D cepstrum. It is also a computationally efficient method. In this article, the 2-D mel-cepstrum-based feature extraction method is applied to the face recognition problem. It should be pointed out that our aim is not to develop a complete face recognition system, but to illustrate the advantages of the 2-D mel cepstrum.

Face recognition is still an active and popular area of research, due to its various practical applications such as security, surveillance, and identification systems. Significant variations in the images of the same faces and slight variations in the images of different faces make it difficult

0091-3286/2010/\$25.00 @ 2010 SPIE to recognize human faces. Feature extraction from facial images is one of the key steps in most face recognition systems. ${ }^{5,6}$

Principal-component analysis (PCA) and linear discriminant analysis (LDA) are well-known techniques that have been used in face recognition. ${ }^{7-9}$ Although LDA is used as a successful dimensional reduction technique in face recognition, direct LDA-based methods cannot provide good performance when there are large variations and illumination changes in the face images. Thus some extensions such as quadratic LDA, ${ }^{10}$ Fisher's LDA, ${ }^{11}$ and direct, exact LDA ${ }^{12}$ have been proposed. PCA is known as a popular linear feature extraction method that is used in one of the most famous techniques, called eigenfaces. In the eigenface method, the image space is simply projected to a lowdimensional feature space. ${ }^{13}$ That is how the dimensional reduction is achieved.

In the 2-D mel cepstrum, the logarithmic division of the 2-D discrete Fourier transform (DFT) grid provides the dimensionality reduction. This is also an intuitively valid representation in that most natural images are lowpass in nature. Unlike the Fourier or discrete cosine transform (DCT) domain features, high-frequency DFT and DCT coefficients are not discarded in an ad hoc manner. They are simply combined in bins of frequency values in a logarithmic manner during the 2-D mel-cepstrum computation.

The rest of the paper is organized as follows. In Sec. 2, the proposed 2-D mel-cepstrum-based feature extraction method is described. In Sec. 3, the support vector machine, a well-known classification method, is briefly explained. The 2-D mel-cepstrum matrices obtained from facial images are converted into vectors and classified using the SVM, which has been successfully used in face recognition applications. ${ }^{14-16}$ In Sec. 4, experimental results are presented. 


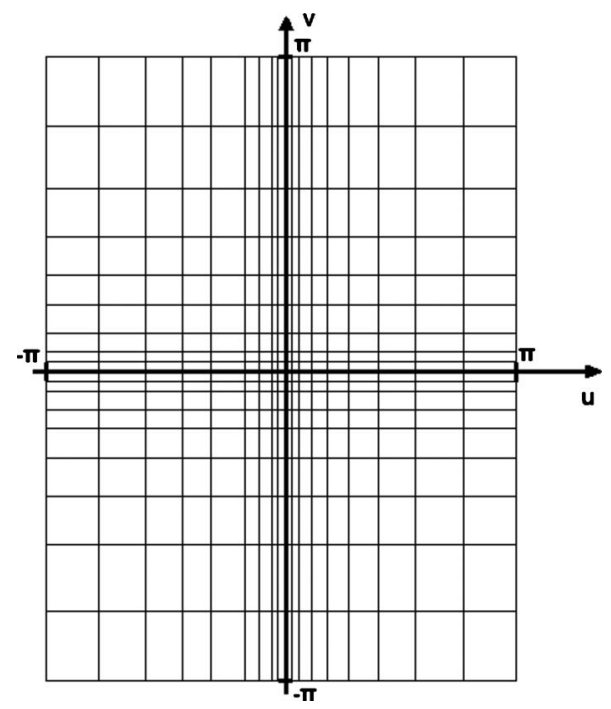

Fig. 1 A representative 2-D mel-cepstrum grid in the DTFT domain. Cell sizes are smaller at low frequencies than at high frequencies.

\section{The 2-D Mel-Cepstrum Feature Extraction}

The 2-D cepstrum has been used for shadow detection, echo removal, automatic intensity control, enhancement of repetitive features, and cepstral filtering. ${ }^{1-3}$ In this article, the 2-D mel cepstrum is used for representing images or image regions.

The 2-D cepstrum $\hat{y}(p, q)$ of a 2-D image $y\left(n_{1}, n_{2}\right)$ is defined as follows:

$\hat{y}(p, q)=F_{2}^{-1}\left(\log \left(|Y(u, v)|^{2}\right)\right)$,

where $(p, q)$ denotes 2-D cepstral quefrency coordinates, $F_{2}^{-1}$ denotes 2-D inverse discrete-time Fourier transformation (IDTFT), and $Y(u, v)$ is the 2-D discrete-time Fourier transform (DTFT) of the image $y\left(n_{1}, n_{2}\right)$. In practice, the FFT algorithm is used to compute the DTFT.

In the 2-D mel cepstrum the DTFT domain data are divided into nonuniform bins in a logarithmic manner as shown in Fig. 1, and the energy $|G(m, n)|^{2}$ of each bin is computed as follows:

$|G(m, n)|^{2}=\sum_{k, l \in B(m, n)}|Y(k, l)|^{2}$,

where $Y(k, l)$ is the discrete fourier transform (DFT) of $y\left(n_{1}, n_{2}\right)$, and $B(m, n)$ is the $(m, n)$ th bin of the logarithmic grid. Bin sizes are smaller at low frequencies than at high frequencies. This approach is similar to the mel-cepstrum computation in speech processing. Like speech signals, most natural images including face images are lowpass in nature. Therefore, there is more signal energy at low frequencies than at high frequencies. Logarithmic division of the DFT grid emphasizes high frequencies. After this step, 2-D mel-frequency cepstral coefficients $\hat{y}_{m}(p, q)$ are computed using either inverse DFT or DCT as follows:

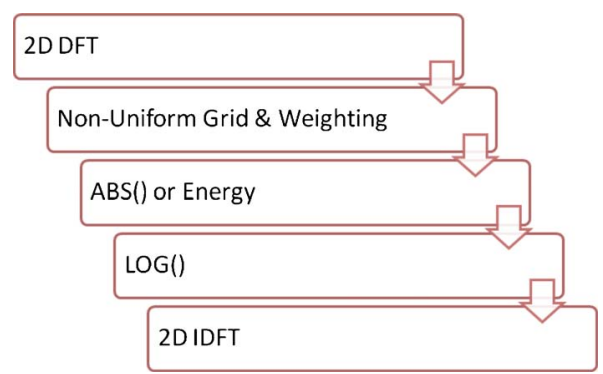

Fig. 2 2-D-cepstrum-based feature extraction algorithm.

$\hat{y}_{m}(p, q)=F_{2}^{-1}\left(\log \left(|G(m, n)|^{2}\right)\right)$.

The size of the inverse DFT (IDFT) is smaller than the size of the forward DFT used to compute $Y(k, l)$, because of the logarithmic grid shown in Fig. 1. It is also possible to apply different weights to different bins to emphasize certain bands, as in speech processing. Since several DFT values are grouped together in each bin, the resulting 2-D melcepstrum sequence computed using the IDFT has smaller dimensions than the original image. The steps of the 2D mel-cepstrum based feature extraction scheme are summarized below:

- The $N$-by- $N$ 2-D DFTs of face images are calculated. The DFT size $N$ should be larger than the image size. It is better to select $N=2^{r}>\operatorname{dimension}\left(y\left(n_{1}, n_{2}\right)\right)$ to take advantage of the FFT algorithm during DFT computation.

- The nonuniform DTFT grid is applied to the resulting DFT matrix, and the energy $|G(m, n)|^{2}$ of each bin is computed. Each bin of the grid can be also weighted with a coefficient. The new data size is $M$ by $M$, where $M \leq N$.

- The logarithms of the bin energies $|G(m, n)|^{2}$ are computed.

- The 2-D IDFT or 2-D IDCT of the $M$-by- $M$ data is computed to get the $M$-by- $M$ mel-cepstrum sequence.

The flow diagram of the 2-D cepstrum feature extraction technique is given in Fig. 2.

In a face image, edges and facial features generally contribute to high frequencies. In order to extract better representative features, high-frequency component cells of the 2-D DFT grid are multiplied with higher weights than are low-frequency ones in the grid. As a result, high-frequency components are further emphasized.

Invariance of the cepstrum to pixel amplitude changes is an important feature. In this way, it is possible to achieve robustness to illumination invariance. Let $Y(u, v)$ denote the 2-D DTFT of a given image matrix $y\left(n_{1}, n_{2}\right)$. Then $c y\left(n_{1}, n_{2}\right)$ has a DTFT $c Y(u, V)$ for any real constant $c$. The $\log$ spectrum of $c Y(u, V)$ is given as follows:

$\log (|c Y(u, v)|)=\log (|c|)+\log (|Y(u, v)|)$,

and the corresponding cepstrum is 


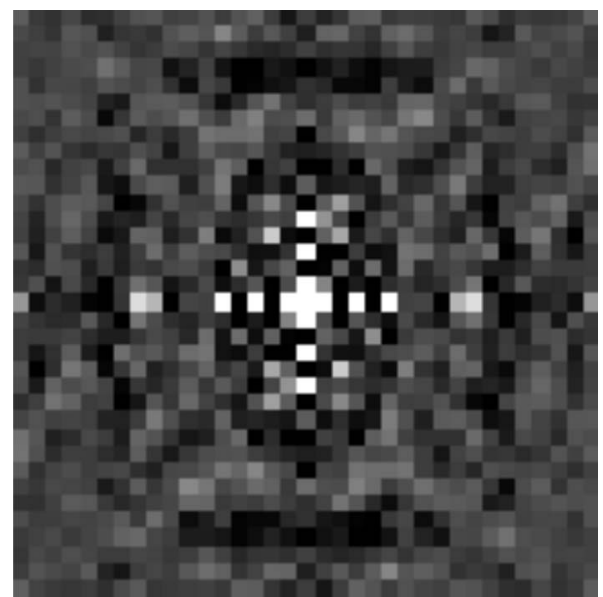

Fig. 3 Magnitude of 35-by-35 2-D mel cepstrum of a face image matrix.

$\psi(p, q)=\hat{a} \delta(p, q)+\hat{y}(p, q)$,

where $\delta(p, q)=1$ for $p=q=0$ and $\delta(p, q)=0$ otherwise. Therefore, the cepstrum values except at $(0,0)$ (the dc term) do not vary with the amplitude changes. Since the Fourier transform magnitudes of $y\left(n_{1}, n_{2}\right)$ and $y\left(n_{1}-k_{1}, n_{2}-k_{2}\right)$ are the same, the 2-D cepstrum and mel cepstrum are shiftinvariant features.

Another important characteristic of the 2-D cepstrum is symmetry with respect to $\hat{y}\left[n_{1}, n_{2}\right]=\hat{y}\left[-n_{1},-n_{2}\right]$. As a result, half of the 2-D cepstrum or $M \times M$ 2-D mel-cepstrum coefficients are enough when IDFT is used.

In this paper, the dimension of the 2-D mel-cepstrum matrix is selected as $M=49,39,35,29$ to represent varioussize face images. Due to the different levels of quantization in logarithmic bins, the size of the 2-D mel-cepstrum matrices varies. The 35-by-35 2-D mel cepstrum of a face image is displayed in Fig. 3. The symmetric structure of the 2-D mel-cepstrum features can be observed in the figure.

In order to emphasize the high-frequency component cells of the 2-D DFT grid further, the normalized weights are organized as in Fig. 4. White values correspond to 1, and black values correspond to 0 . The smallest value used in Fig. 4 is 0.005 .

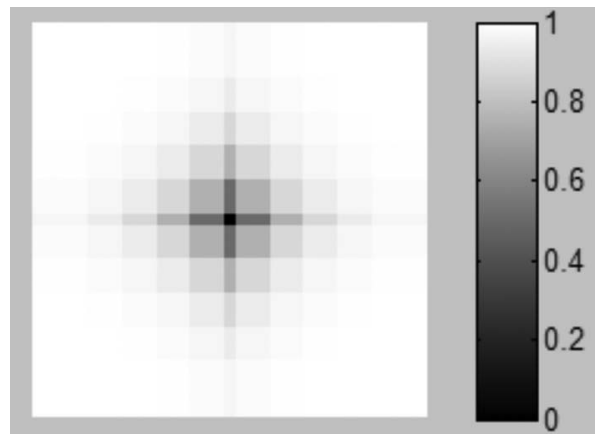

Fig. 4 35-by-35 normalized weights for emphasizing high frequencies.

\section{Feature Classification: Multi-Class SVM}

In this article, a multi-class SVM is used in feature classification. The SVM needs a matrix-to-vector conversion process to convert the 2-D mel-cepstrum matrices to vectors.

The SVM is a supervised machine learning method based on statistical learning theory and developed by Vladimir Vapnik. ${ }^{17}$ The method constructs a hyperplane or a set of hyperplanes in a high-dimensional space that can be used in classification tasks. In this work, an SVM with a multi-class classification support ${ }^{18}$ with radial basis function $(\mathrm{RBF})$ kernel is used. The multi-class classification method uses a one-against-one strategy. ${ }^{19}$

2-D mel-cepstrum matrices are converted to vectors in a raster scan approach before training and classification. The raster scan starts with $\hat{y}_{m}(0,0)$. If there are pixel intensity variations, $\hat{y}_{m}(0,0)$ is ignored and the scan starts from $\hat{y}_{m}(0,1)$.

\section{Experimental Results}

\subsection{Databases}

In this paper, the AR Face Database, ${ }^{20}$ ORL Face Database, ${ }^{21}$ and Yale Face Database ${ }^{22}$ are used.

The AR database, created by Aleix Martinez and Robert Benavente, contains 4000 facial images of 126 subjects. Of these subjects, 70 are male, and the remaining 56 are female. Each subject has different poses, including different facial expressions, illumination conditions, and occlusions (sunglasses and scarf). The face images are all of dimensions 768 by 576 pixels. In this work, 14 non-occluded poses of 50 subjects are used. Face images are converted to grayscale, normalized, and cropped to size $100 \times 85$. Sample poses for randomly selected subjects from the AR database are shown in Fig. 5.

The second database used in this work is the ORL database. It contains 40 subject, and each subject has 10 poses. The images are captured at different time periods, in different lighting conditions, and with different accessories for some of the subjects. In this work 9 poses of each subject are used. In the ORL database, images are all in grayscale with dimensions $112 \times 92$. Sample images from the ORL database are shown in Fig. 6.

The last database used in this work is the Yale database. It contains grayscale facial images of size $152 \times 126$. The database contains 165 facial images belonging to 15 subjects. Each pose of the subjects has a different facial expression and illumination. Sample images from the Yale database are shown in Fig. 7.

\subsection{Procedure and Experimental Work}

In order to compare the performance of various features, 2-D mel-cepstrum-based features, actual image pixel matrices, 2-D-PCA-based features, and 2-D-PCA-based features of illumination-compensated images are applied to multiclass SVM as inputs.

For the purpose of achieving robustness in recognition results, the leave-one-out procedure is used. Let $p$ denote the number of poses for each person in a database. In the test part of the classifier, one pose of each person is used 


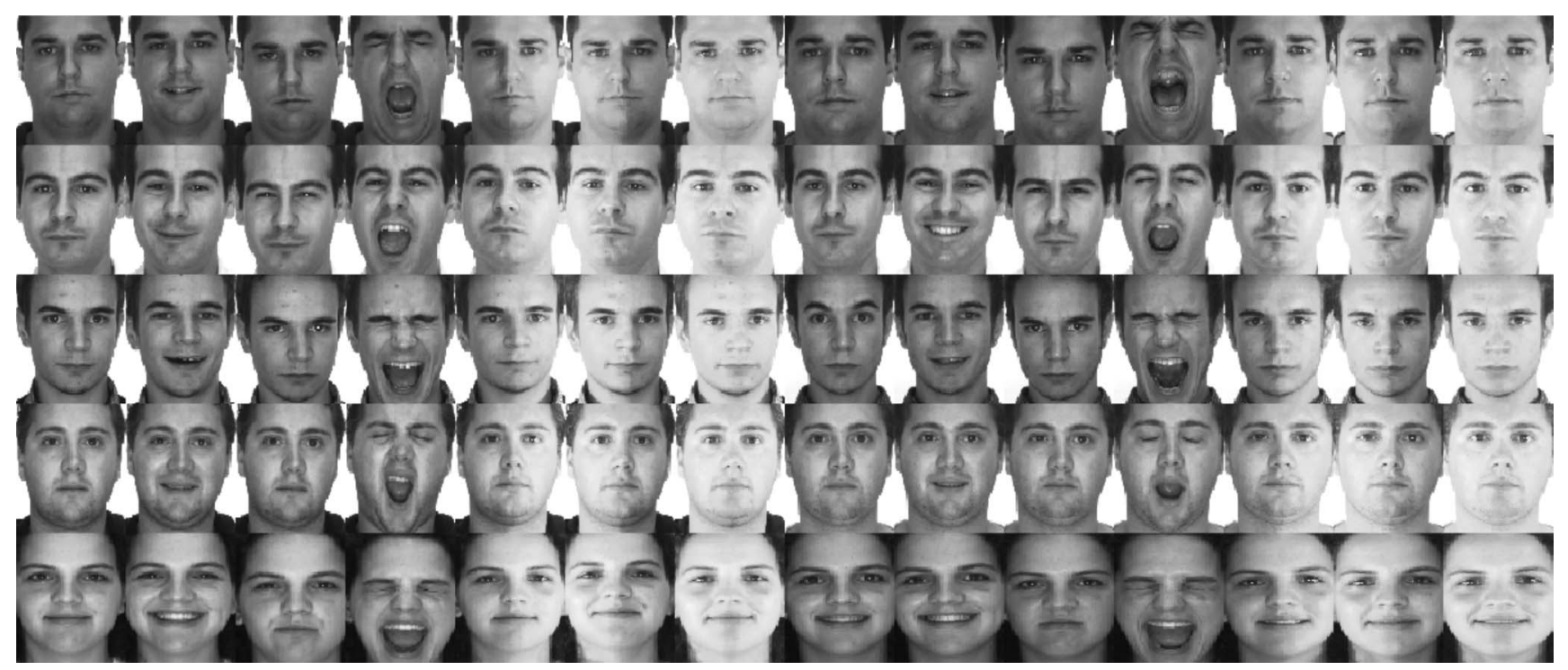

Fig. 5 Sample images for randomly selected subjects from the AR Face Database.

for testing. The remaining $p-1$ poses for each person are used in the training part of the classifier. In the leave-oneout procedure, the test pose is changed in turn and the algorithm is trained with the newly remaining $p-1$ images.
At the end, a final recognition rate is obtained by averaging the recognition rates for each selection of the test pose.

In the extraction of the 2-D mel-cepstrum features, different nonuniform grids are used. Due to these differences,

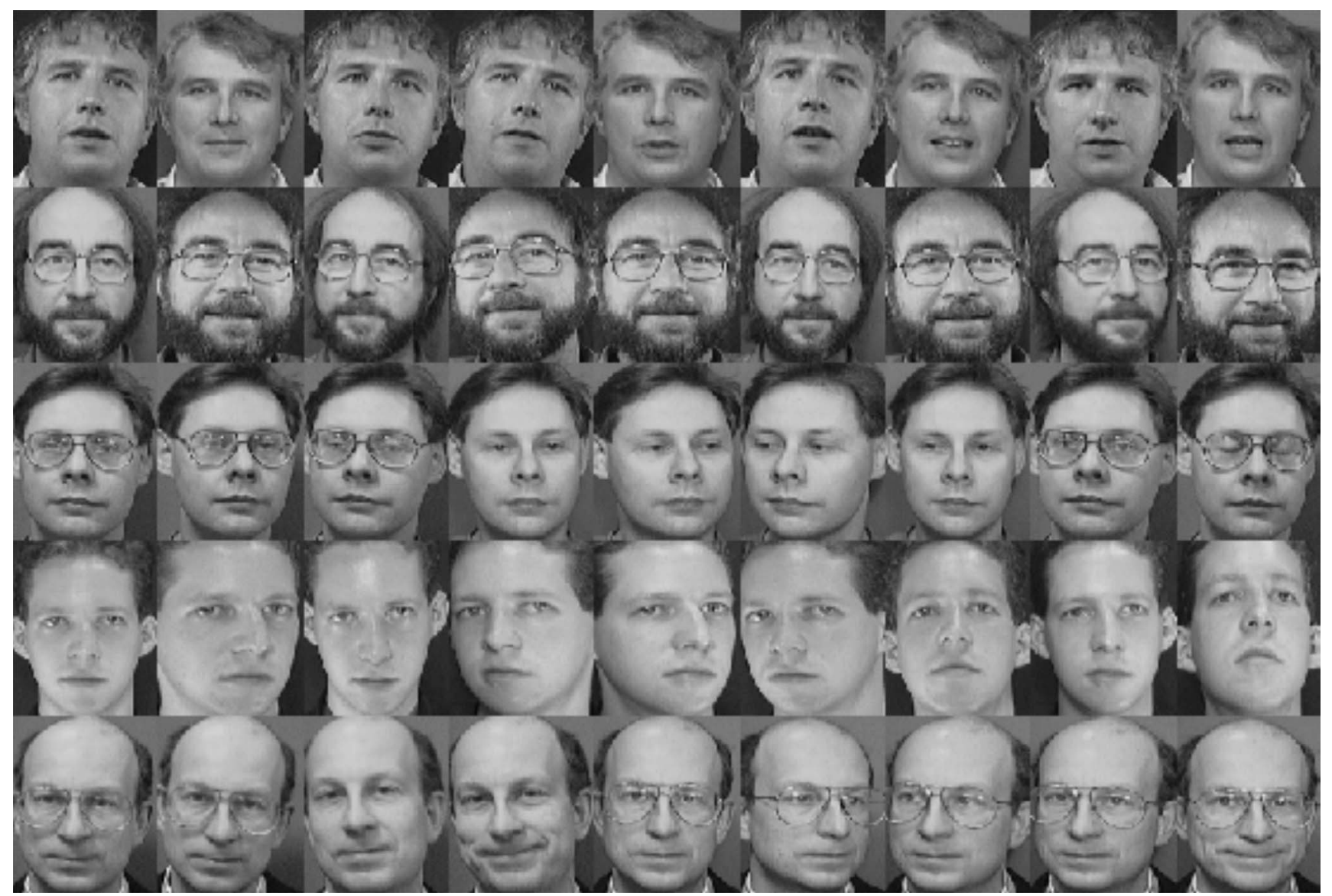

Fig. 6 Sample images for randomly selected subjects from the ORL Face Database. 


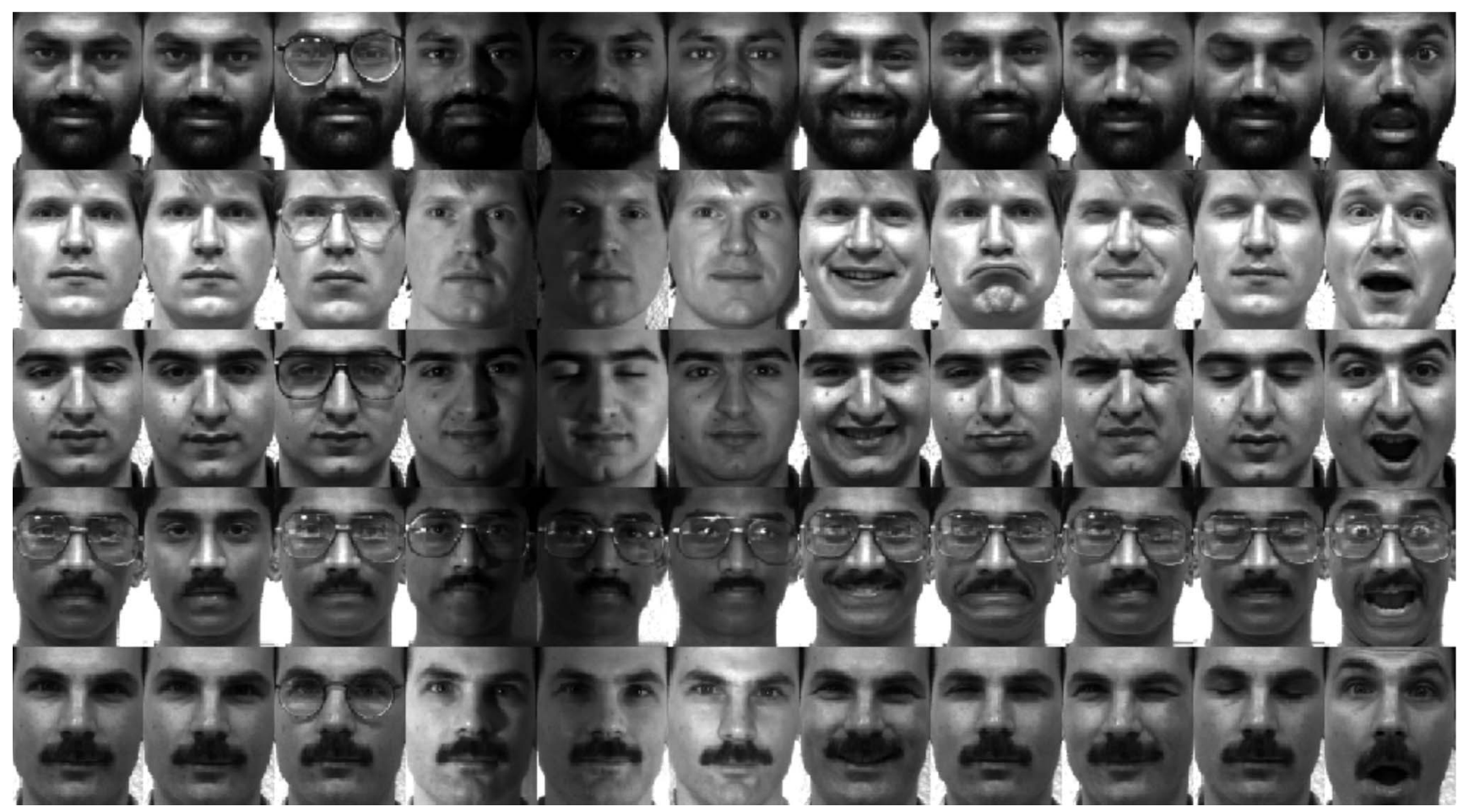

Fig. 7 Sample images for randomly selected subjects from the Yale Face Database.

new 2-D mel-cepstrum features are generated in different dimensions. The 2-D mel-cepstrum features giving the best performance are used in the comparison with the 2-D PCA features and actual image matrices.

Due to the invariance of 2-D mel-cepstral features to pixel amplitude changes, the 2-D mel cepstrum can cope with large illumination changes in a data set. In order to observe the robustness of the mel-cepstral method to illumination variations, a second experiment was carried out. An illumination compensation algorithm (LogAbout method $\left.^{23}\right)$ is implemented and used as a preprocessing step before extracting features using 2-D PCA. The illumination-compensated image samples of each database are displayed in Figs. 8-10, respectively.

\subsubsection{AR Face Database}

In Table 1, the average recognition rate of each leave-oneout step is given for the SVM classifier when different 2-D mel-cepstrum features are used. 2D mel-cepstrum features

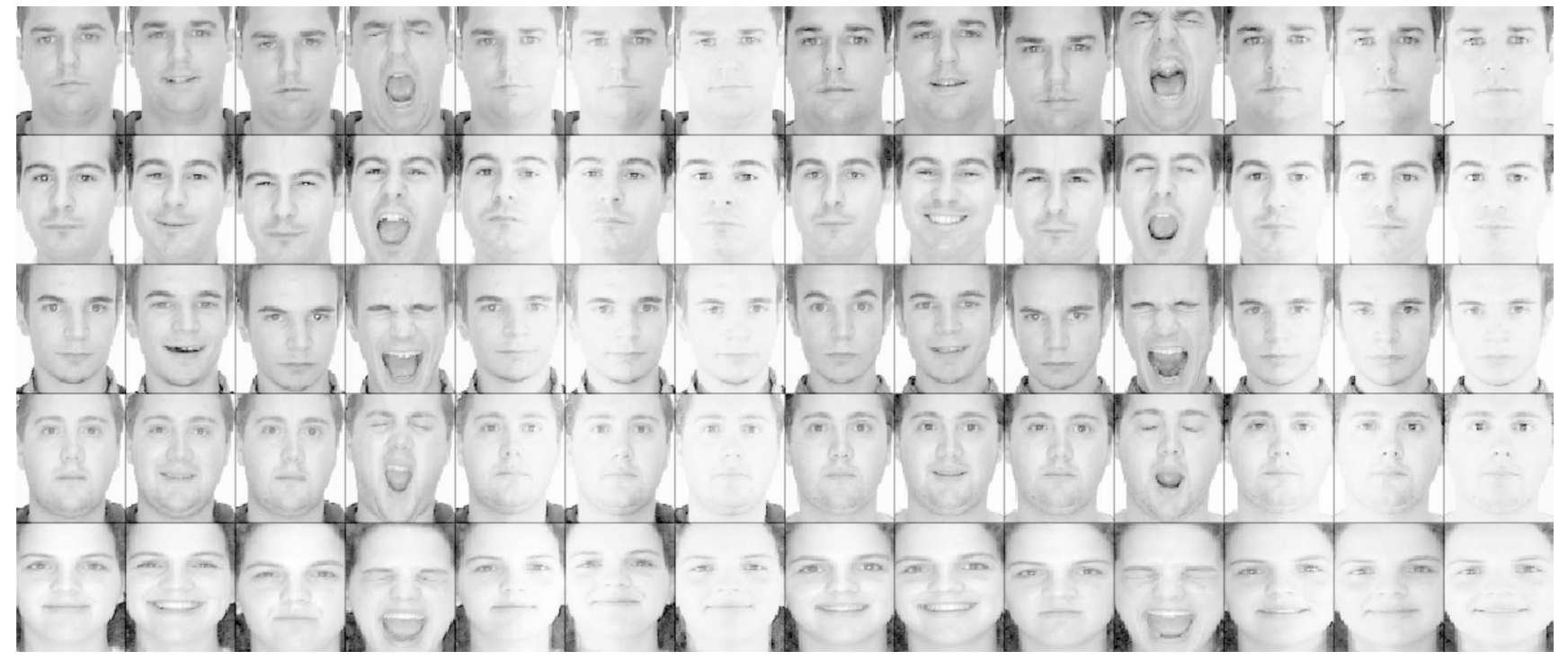

Fig. 8 Illumination-compensated sample images for randomly selected subjects from the AR Face Database. 


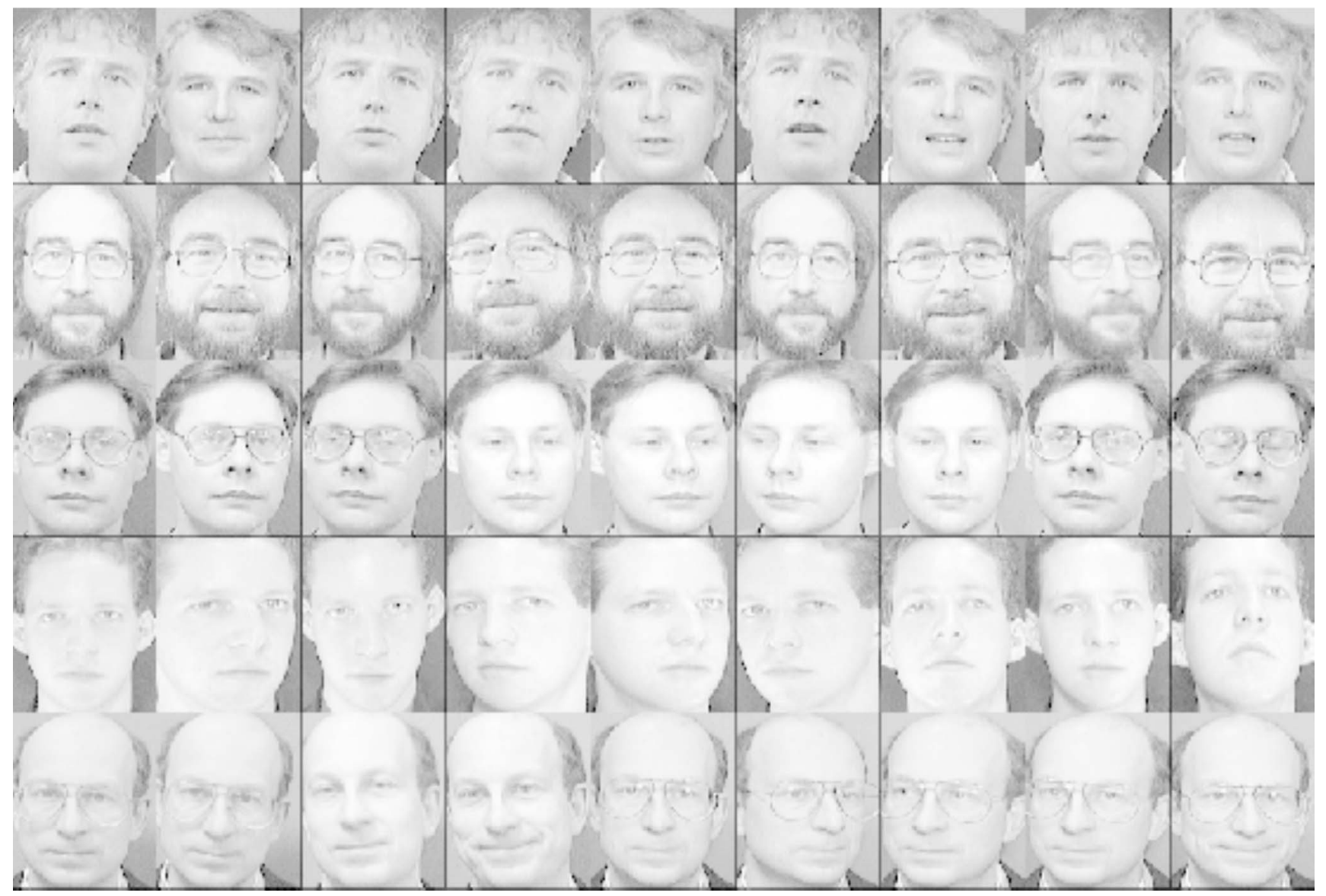

Fig. 9 Illumination-compensated sample images for randomly selected subjects from the ORL Face Database.

outperform the PCA, PCA with illumination correction, and actual image pixel-value-based features, as shown in Table 2 in all three databases, including the AR Face Database.

\subsubsection{ORL Face Database}

In the ORL Face Database, recognition experiments repeated by using different DTFT domain grids in the calculation of 2-D mel-cepstrum features. These features are applied to multi-class SVM, and the recognition rates are shown in the Table 1.

\subsubsection{Yale Face Database}

The Yale Face Database contains images captured under different illumination conditions. Since there exist illumination changes in the database, we simply set the $(0,0)$ value of the 2-D mel cepstrum $\hat{y}(0,0)=1$ in all cases to normalize illumination changes. As a result, the average recognition rate of the database increased from $93.94 \%$ to 98.18\% when the multi-class SVM-based classifier was used. The performance of different-sized 2-D mel-cepstrum features using Yale Face Database images are presented in Table 1.

Our final average recognition rate of $98.18 \%$ is higher than the ones reported in Ref. 24-27 for the Yale Face Database. Reported recognition rates are $97.33 \%, 96.00 \%$, $92.73 \%$, and $89.09 \%$ in those references, respectively.
Based on the recognition rates presented in the Table 1, grid 2 provides better representative features in the AR Face Database. Grid 4 provides an increase in recognition rate when the ORL Face Database is used. In the Yale Face Database, cepstral features extracted using grid 1 provide better performance than other nonuniform grids. Therefore, the grid that performs best in a face database is used as the nonuniform DTFT domain grid in the extraction of 2-D mel-cepstrum features in the comparison of performance with the other feature extraction techniques.

The performance of actual image pixel matrices and of feature matrices based on 2-D PCA, 2-D PCA with illumination correction, and 2-D mel-cepstrum are tested by converting these matrices to feature vectors and applying these feature vectors to the multi-class SVM. The recognition rate for each database is displayed in Table 2 for each feature extraction technique.

Based on the experiments described, the 2-D PCA features do not provide better results than the proposed 2-D mel-cepstrum features. Moreover, the of 2-D PCA features have higher computational complexity than 2-D cepstrum features, which are computed using FFT. The cost of computing a 2-D mel-cepstrum sequence for an $N$-by- $N$ image is $O\left(N^{2} \log (N)+M^{2} \log (M)\right)$ and an additional $M^{2} / 2$ logarithm computations, which can be implemented using a lookup table. Another advantage of the 2-D mel-cepstrum 


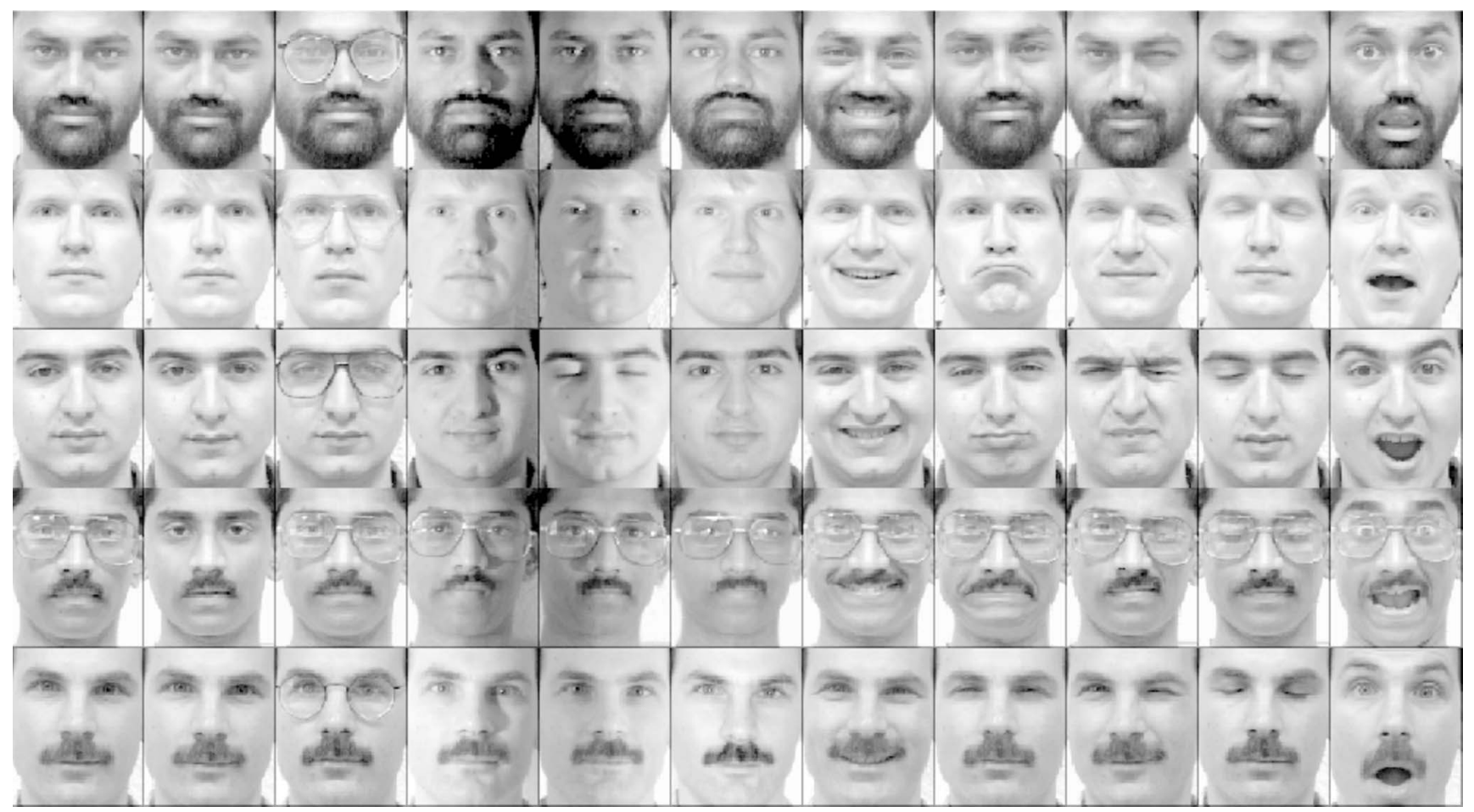

Fig. 10 Illumination-compensated sample images for randomly selected subjects from the Yale Face Database.

Table 1 Recognition rates (RR) of face databases using 2-D melcepstrum features when different DTFT domain grids are used.

\begin{tabular}{ccccc}
\hline \hline & & \multicolumn{3}{c}{$\begin{array}{c}\text { RR of face database } \\
(\%)\end{array}$} \\
\cline { 3 - 5 } Grid & Feature size & AR & ORL & Yale \\
\hline 1 & 1225 & 98.71 & 98.05 & 98.18 \\
2 & 780 & 98.71 & 98.61 & 96.96 \\
3 & 630 & 98.71 & 98.61 & 96.96 \\
4 & 435 & 98.42 & 99.16 & 96.96 \\
\hline \hline
\end{tabular}

is its invariance to illumination variations. Even 2-D PCA illumination-compensated (LogAbout method) images cannot perform better than the proposed 2-D mel-cepstrum features in image representation and elimination of illumination changes.

\section{Conclusion}

In this article, a 2-D mel-cepstrum-based feature extraction technique is proposed for image representation. Invariance to amplitude changes and translational shifts are important properties of the 2-D mel-cepstrum and 2-D cepstrum. Features based on the 2-D mel-cepstrum provide not only better recognition rates but also dimensionality reduction in feature matrix sizes in the face recognition problem. Our experimental studies indicate that the 2-D mel-cepstrum

Table 2 Recognition rates (RR) of SVM-based classifier with different feature sets. IC: illumination compensation.

\begin{tabular}{|c|c|c|c|c|c|c|}
\hline \multirow[b]{2}{*}{ Features } & \multicolumn{2}{|c|}{ AR } & \multicolumn{2}{|c|}{ ORL } & \multicolumn{2}{|c|}{ Yale } \\
\hline & $\begin{array}{l}\text { RR } \\
(\%)\end{array}$ & $\begin{array}{l}\text { Vector } \\
\text { size }\end{array}$ & $\begin{array}{l}\text { RR } \\
(\%)\end{array}$ & $\begin{array}{l}\text { Vector } \\
\text { size }\end{array}$ & $\begin{array}{l}\text { RR } \\
(\%)\end{array}$ & $\begin{array}{c}\text { Vector } \\
\text { size }\end{array}$ \\
\hline Original images & 96.85 & 8500 & 98.05 & 10304 & 88.00 & 19152 \\
\hline 2-D PCA & 96.85 & 1200 & 98.33 & 1680 & 87.87 & 1368 \\
\hline 2-D PCA with IC algorithm & 97.28 & 1200 & 98.88 & 1680 & 89.09 & 1368 \\
\hline Proposed 2-D mel cepstrum & 98.71 & 780 & 99.16 & 435 & 98.18 & 1225 \\
\hline
\end{tabular}


method is superior to classical baseline feature extraction methods in facial image representation with regard to computational complexity.

\section{References}

1. B. Ugurur Töreyin and A. Enis Çetin, "Shadow detection using 2D cepstrum," in Acquisition, Tracking, Pointing, and Laser Systems Technologies XXIII, Proc. SPIE 7338, 733809 (2009).

2. Y. Yeshurun and E. L. Schwartz, "Cepstral filtering on a columnar image architecture: a fast algorithm for binocular stereo segmentation," IEEE Trans. Pattern Anal. Mach. Intell. 11, 759-767 (Jul. 1989).

3. J. K. Lee, M. Kabrisky, M. E. Oxley, S. K. Rogers, and D. W. Ruck, "The complex cepstrum applied to two-dimensional images," Pattern Recogn. 26, 1579-1592 (1993).

4. A. Enis Çetin and R. Ansari, "Convolution-based framework for signal recovery and applications," J. Opt. Soc. Am. A 5, 1193-1200 (1988).

5. W. Zhao, R. Chellappa, A. Rosenfeld, and P. J. Phillips, "Face recognition: a literature survey," ACM Comput. Surv. 35, 399-458 (2003).

6. R. Brunelli and T. Poggio, "Face recognition: features versus templates," IEEE Trans. Pattern Anal. Mach. Intell. 15, 1042-1052 (1993).

7. K. Etemad and R. Chellappa, "Discriminant analysis for recognition of human face images," J. Opt. Soc. Am. A 14, 1724-1733 (1997).

8. L.-F. Chen, H.-Y. M. Liao, M.-T. Ko, J.-C. Lin, and G.-J. Yu, "A new LDA-based face recognition system which can solve the small sample size problem," Pattern Recogn. 33, 1713-1726 (2000).

9. A. M. Martinez and A. C. Kak, "PCA versus LDA," IEEE Trans. Pattern Anal. Mach. Intell. 23, 228-233 (Feb. 2001).

10. J. Lu, K. N. Plataniotis, and A. N. Venetsanopoulos, "Regularized discriminant analysis for the small sample size problem in face recognition," Pattern Recogn. Lett. 24, 3079-3087 (2003).

11. P. N. Belhumeur, J. P. Hespanha, and D. J. Kriegman, "Eigenfaces vs. fisherfaces: recognition using class specific linear projection," IEEE Trans. Pattern Anal. Mach. Intell. 19, 711-720 (Aug. 1997), http:// dx.doi.org/10.1109/34.598228.

12. H. Yu, "A direct LDA algorithm for high-dimensional data with application to face recognition," Pattern Recogn. 34, 2067-2070 (Oct. 2001).

13. M. Turk and A. Pentland, "Eigenfaces for recognition," J. Cogn Neurosci. 3, 71-86 (Jan. 1991)

14. J. Qin and Z.S. He "A SVM face recognition method based on Gabor-featured key points," in Proc. 2005 Int. Conf. on Machine Learning and Cybernetics, Vol. 8, pp. 5144-5149 (2005).

15. G. Dai and C. Zhou, "Face recognition using support vector machines with the robust feature," in Proc. 12th IEEE Int. Workshop on Robot and Human Interactive Communication, ROMAN 2003, pp. 49-53 (2003).

16. G. Guo, S. Z. Li, and K. Chan, "Face recognition by support vector machines," in FG '00: Proc. Fourth IEEE Int. Conf. on Automatic Face and Gesture Recognition 2000, p. 196, IEEE Computer Soc., Washington (2000).

17. B. E. Boser, I. M. Guyon, and V. N. Vapnik, "A training algorithm for optimal margin classifiers," in COLT '92: Proc. Fifth Annual Workshop on Computational Learning Theory, pp. 144-152, ACM, New York (1992).

18. C. C. Chang and C. J. Lin, LIBSVM: A Library for Support Vector Machines, http://www.csie.ntu.edu.tw/ cjlin/libsvm (2001)

19. S. Knerr, L. Personnaz, and G. Dreyfus, "Single-layer learning revisited: a stepwise procedure for building and training a neural network," in Neurocomputing: Algorithms, Architectures and Applications, J. Fogelman, Ed., Springer-Verlag (1990).

20. A. M. Martinez and R. Benavente, "The AR face database," CVC Tech. Report 24 (1998).

21. F. S. Samaria and A. C. Harter, "Parameterisation of a stochastic model for human face identification," in Proc. Second IEEE Workshop on Applications of Computer Vision, pp. 138-142 (1994).

22. Yale Univ., "Yale Face Database," http://cvc.yale.edu/projects/ yalefaces/yalefaces.html (1997).

23. H. Liu, W. Gao, J. Miao, D. Zhao, G. Deng, and J. Li, "Illumination compensation and feedback of illumination feature in face detection," in Proc. ICII Beijing Int. Conf. on Info-tech and Info-net, Vol. 3, pp. 444-449 (2001).

24. H. Cevikalp, M. Neamtu, M. Wilkes, and A. Barkana, "Discriminative common vectors for face recognition," IEEE Trans. Pattern Anal. Mach. Intell. 27, 4-13 (2005).

25. Y. Wu, K. Luk Chan, and L. Wang, "Face recognition based on discriminative manifold learning," in Proc. 17th Int. Conf. on Pattern Recognition (ICPR'04), Vol. 4, pp. 171-174, IEEE Computer Soc., Washington (2004)

26. X. Qiu, W. Wang, J. Song, X. Zhang, and S. Liu, "Face recognition based on binary edge map and support vector machine," in 1st Int. Conf. on Bioinformatics and Biomedical Engineering, ICBBE 2007, pp. 519-522 (2007).

27. K. Tan and S. Chen, "Adaptively weighted sub-pattern PCA for face recognition," Neurocomputing 64, 505-511 (2005).

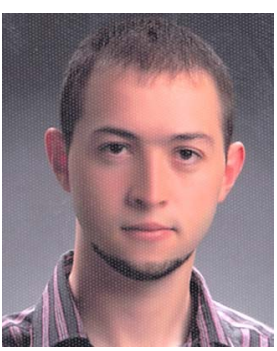

Serdar Çakır received his BS degree from Eskisehir Osmangazi University in 2008. Immediately after graduation he joined Bilkent University, where currently he is a teaching assistant in the Electrical and Electronics Engineering Department. Currently, he is a MSc candidate at Bilkent University under the supervision of Prof. A. Enis Cetin. His main research interests are image processing and pattern recognition.

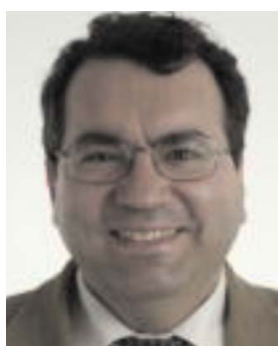

A. Enis Çetin studied electrical engineering at the Middle East Technical University. After getting his BSc degree, he got his MSE and $\mathrm{PhD}$ degrees in systems engineering from the Moore School of Electrical Engineering at the University of Pennsylvania in Philadelphia. Between 1987 and 1989, he was an assistant professor of electrical engineering at the University of Toronto, Canada. Since then he has been with Bilkent University, Ankara, Turkey. Currently he is a full professor. During the summers of 1988, 1991, and 1992 he was with Bell Communications Research (Bellcore), New Jersey. He spent the 1996-1997 academic year at the University of Minnesota, Minneapolis, USA, as a visiting associate professor. Prof. Çetin is a Fellow of the IEEE. He was an associate editor of the IEEE Transactions on Image Processing between 1999 and 2003, and a member of the Signal Processing Theory and Methods Technical Committee of the IEEE Signal Processing Society. He is currently on the editorial board of the EURASIP Journal of Applied Signal Processing and the Journal of Machine Vision and Applications. He was the co-chair of the IEEE-EURASIP Nonlinear Signal and Image Processing Workshop (NSIP'99), which was held in June 1999 in Antalya, Turkey. He was also the technical co-chair of the European Signal Processing Conference, EUSIPCO-2005. He is the scientific committee member of the European Commission-funded FP7 project FIRESENSE. 